\title{
DEVELOPMENT OF MECHANIZED FEEDING SYSTEM WITH COMPUTATIONAL ANALYSIS
}

\author{
Dheeraj Kumar ${ }^{1}$, Divyansh Agarwal ${ }^{2}$, Govind Maheshwari ${ }^{3}$, Pradeep Khanna ${ }^{4}$ \\ ${ }^{1}$ Student, Netaji Subhas Institute of Technology (India) \\ ${ }^{2}$ Student, Netaji Subhas Institute of Technology (India) \\ ${ }^{3}$ Student, Netaji Subhas Institute of Technology (India) \\ ${ }^{4}$ Associate Professor, Department of MPAE, Netaji Subhas Institute of Technology (India)
}

\begin{abstract}
The successful assembly lines in mass production require performance based part feeding systems. However, the lack of flexible programmable part positioners, facilitating the handling of smart sized parts, restricts the efficiency of a production unit. So, a flexible feeder in batch production is indeed important. This can dramatically reduce the time needed to bring new products to market and permit cost of assembly lines to be used for various products. This project aims to develop a mechanized segment feeder with the help of a prototype for the same. The system consists of two wedges for part loading and two sliders along with a motor to drive these sliders. The slider speed may be changed as per convenience. To study the performance of the system, wood is majorly used for wedges and sliders, pinion and driving gear are used to drive the slider and plastic workpiece of circular cross-section are used.
\end{abstract}

Keywords: Assembly line; Flexible

\section{INTRODUCTION}

The predominant approach today to introduce factory automated technology into manufacturing is to selectively apply automation and create islands of automation. The phrase "islands of automation" has been used to describe the transition from conventional or mechanical manufacturing to the automated factory. Automated Flexible Assembly Systems (FAS) are capable of being programmed to assemble several different products on a single assembly line with minimal equipment changes. For manufacturers who produce several different products in volumes too small to justify the expense of having a separate assembly line for each product, FASs are more economical than traditional manual or dedicated automated production methods. FASs allow a manufacturer to produce several different products in limited volumes with essentially the same amount of equipment that would be necessary to produce a single product in high production volumes. Flexible assembly systems are also advantageous over dedicated automated systems because when a product is updated or redesigned, a FAS typically can be modified to produce the new, redesigned product with minimal or no equipment changes. In accordance with the above described characteristics, FASs require parts feeding systems which can feed parts in proper orientation; the following project aims at proposing a prototype for solving the above said utility. A typical parts feeding system comprises numerous components. First, as storage means, such as a wedge-shaped box is used to store a plurality of parts. A pair of slider is used which transfers the parts from the storage means to a parts feeder, where orientation of the parts occurs (Fig.1-2). The presented system offers flexibility in the size of cross-section of the prismatic components. The given mechanism is equipped with a long length chute which helps to ensure the orientation of the parts, where the parts flow over the delivery chute under their own weight and even by a push from the other parts. The given system is independent of the environment in which the parts may be given, like even if the parts are lubricated, the mechanism can perform similarly.

The study even includes a study on the performance of the segment feeder, where in the wedge is loaded with a given constant load of parts and a variable controllable speed is given to slider by a motor to determine its performance.

\section{OBJECTIVE}

The study can be divided into two illustrative categories:

- Fabrication and Development of storage wedge and chute for feeding solid cylindrical parts.

- Analysis of operating parameters governing the feed rate of the setup. 


\subsection{Design}

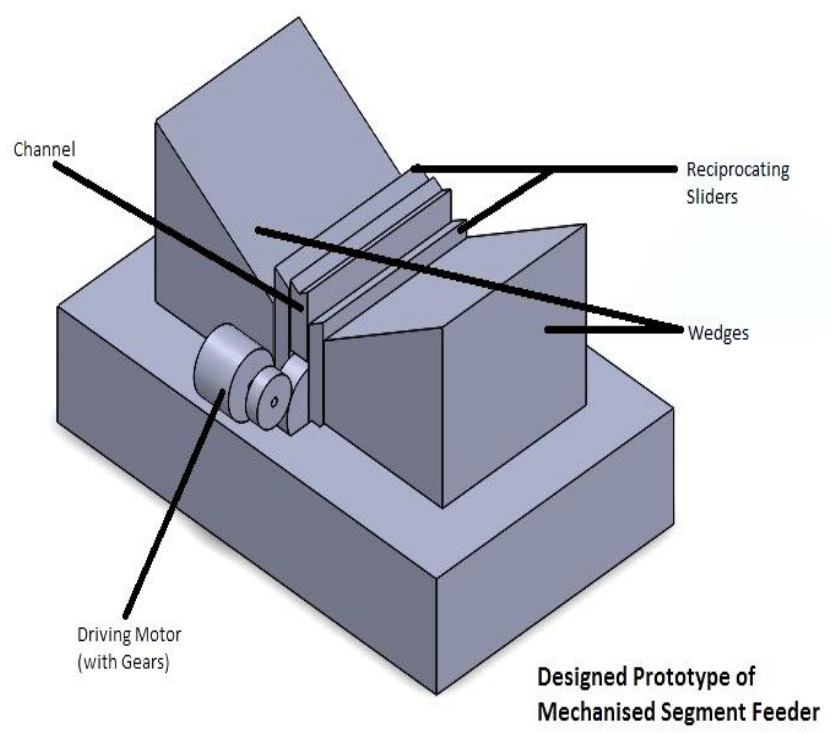

Fig. 1

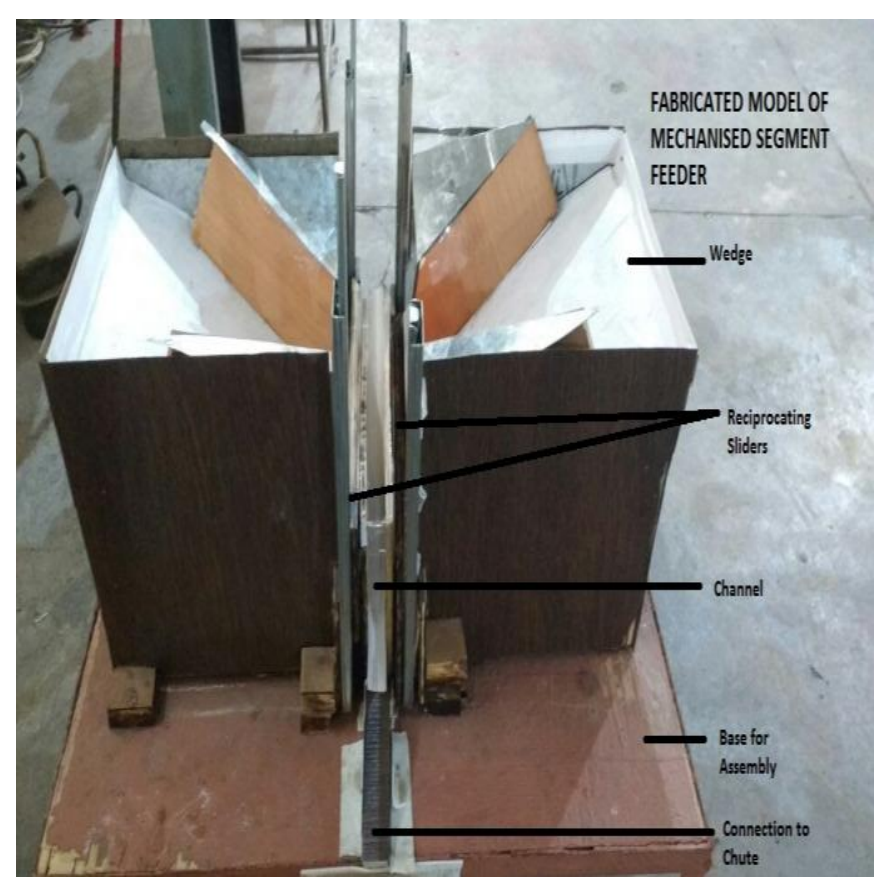

Fig. 2

\subsection{Components of the Setup}

1) Wedge: Two wedges made of wood for storing the parts. Dimensions of the wedge: $250 \mathrm{~mm} * 250 \mathrm{~mm} * 250 \mathrm{~mm}$.

2) Slider: Two sliders made of wood for loading the parts into feeder. Dimensions of the slider: $250 \mathrm{~mm} * 10 \mathrm{~mm} * 100 \mathrm{~mm}$.

3) Channel: A passage through which work pieces are fed out. Dimensions: $250 \mathrm{~mm} * 150 \mathrm{~mm} * 14 \mathrm{~mm}$.

4) Pinion: A plastic gear attached to the motor with diameter:

5) Driving Gear: A plastic gear used for the driving the slider with diameter:
6) Motor: A DC Motor with rated maximum RPM of 100 .

7) Power Source: A Motor Driver Module - Arduino setup was used to supply variable voltage to the motor.

\subsection{Arduino Code for Variable Voltage Supply}

int analogOut $1=10$;

$/ /$ pin

Connection to Motor Driver module.

int analogOut2 = 11;

$/ / \mathrm{pin}$

Connection to Motor Driver module.

int low $=230$;

correspondong to low speed.

int high $=255$;

// Analog Value

correspondong to high speed.

// Analog Value

void $\operatorname{setup}()$

\{

pinMode(analogOut1, OUTPUT); //Pin 10 as Output.

pinMode(analogOut2, OUTPUT); //Pin 11 as Output. \}

void $\operatorname{loop}()\{$

analogWrite(analogOut2, 0);

$/ /$ pin 11 set to

Ground (0 volts).

analogWrite(analogOut1, low); $\quad$ //pin $\quad 10$ assigned analog value LOW.

//Value is changed to HIGH to alter Speed.

delay(5000); $\quad / /$ a 5 second delay

Before Next Loop.

\section{EXPERIMENTAL WORK}

\subsection{Experimental Procedure}

In order to utilize the feeder for various industrial based applications it is important to analyze the feeder in response to different operating factors. The present work is based on graphical analysis of the performance of an elevated feeder. A systematic analysis of the part feed rate of the feeder was carried out under different operating conditions. A series of experiments was carried out by varying these parameters.

The Various parameters which were involved in the analysis are:

1) Part Size: It refers to the diameter of the solid cylindrical components. Length of the components is kept fixed.

2) Slider Speed: It refers to speed of the slider in rpm.

3) Part Population: It refers to the number of components put in the wedges for the analysis.

\subsection{Range of Parameters}

1) Part Size (mm): 6, 8, 10, 12.

2) Slider Speed (rpm): 26, 40, 52, 62, 94

3) Part Population: 50, 75, 100

The experiment was carried out by utilizing the one factor at a time technique, which involved varying one of the parameters while keeping the other governing parameters constant. The feed rate was obtained for each set of operating conditions. 


\section{COMPUTATIONAL ANALYSIS}

This section provides the computational analysis which depicts the variation of feed rate with changes in various operating parameters. Various graph and statistics were obtained using Design Expert 10 - Stat-Ease.

\subsection{Best Fit Equation}

The relation between part population, part size and slider speed to give the desired feed rate was analysed upto sixth order equation, but quadratic was the most appropriate.

\begin{tabular}{|c|c|c|c|c|c|}
\hline \multirow{2}{*}{\multicolumn{6}{|c|}{$\begin{array}{l}\text { Response } 1 \quad \text { Feed Rate Transform: None } \\
\text { t" WARNING: The Cubic Model and higher are Aliased! " }{ }^{\text {th }}\end{array}$}} \\
\hline & & & & & \\
\hline \multicolumn{6}{|c|}{ Summary (detailed tables shown below) } \\
\hline & Sequential & Lack of Fit & Adjusted & Predicted & \\
\hline Source & $\mathrm{p}$-value & p-value & R-Squared & R-Squared & \\
\hline Linear & $<0.0001$ & & 0.8937 & 0.8839 & \\
\hline $2 \mathrm{Fl}$ & 0.2144 & & 0.8967 & 0.8776 & \\
\hline$\underline{\text { Quadratic }}$ & $\leq 0.0001$ & & $\underline{0.9695}$ & $\underline{0.9649}$ & Sugoested \\
\hline Cubic & $<0.0001$ & & 0.9987 & 0.9983 & Aliased \\
\hline
\end{tabular}

Fig. 3
The desired quadratic equation was as follows:

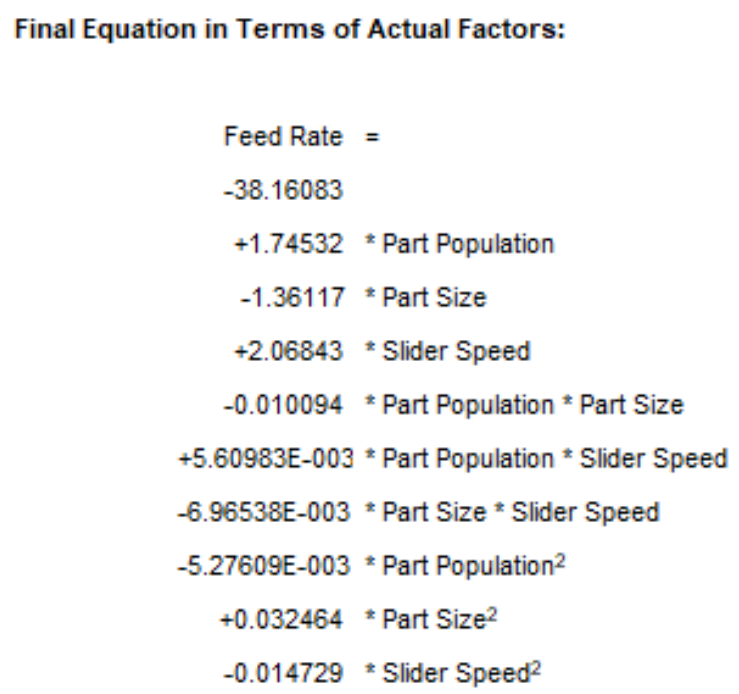

Fig. 4

\subsection{Contour Plot}

The contour plot is a 2-Dimensional representation of the response plotted against combination of numeric factors.
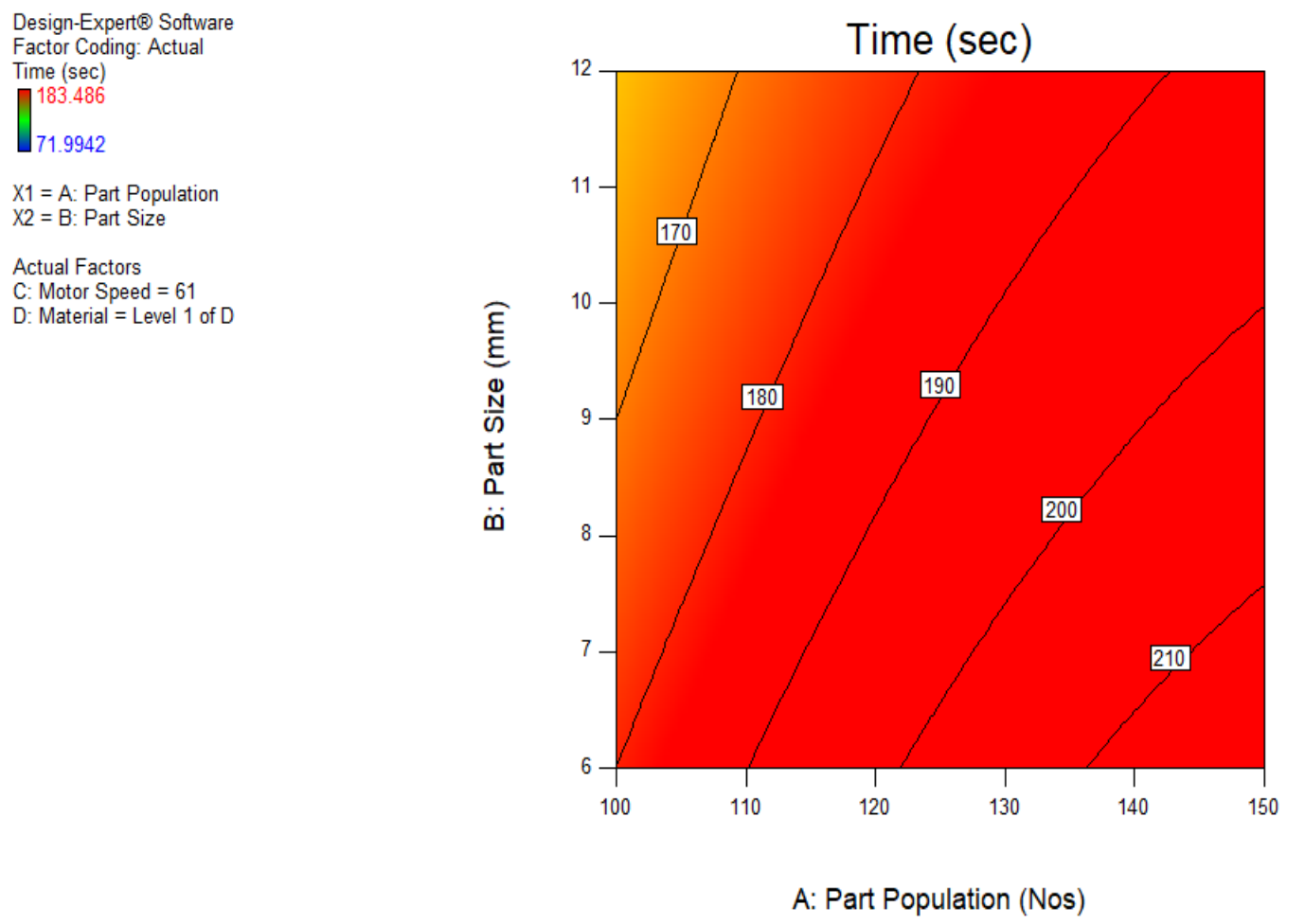

Fig.5 


\subsection{Normal Plot of Residuals}

The normal plot indicates whether the residuals follow a normal distribution, in which the points will follow a straight line.

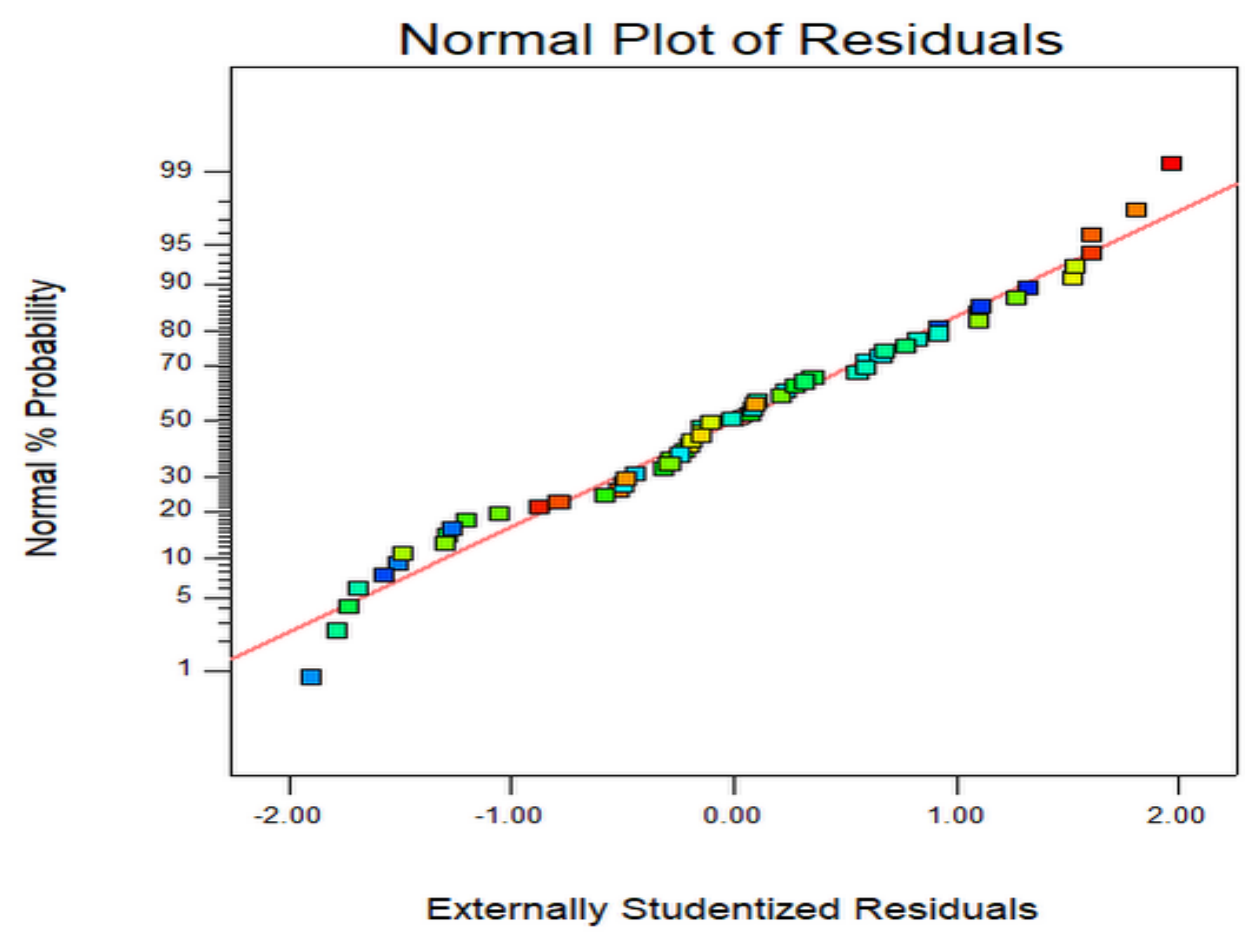

\subsection{Predicted v/s Actual}

Fig. 6

A graph of the observed (actual) response values versus the predicted response values. It helps to detect observations that are not well predicted by the model.

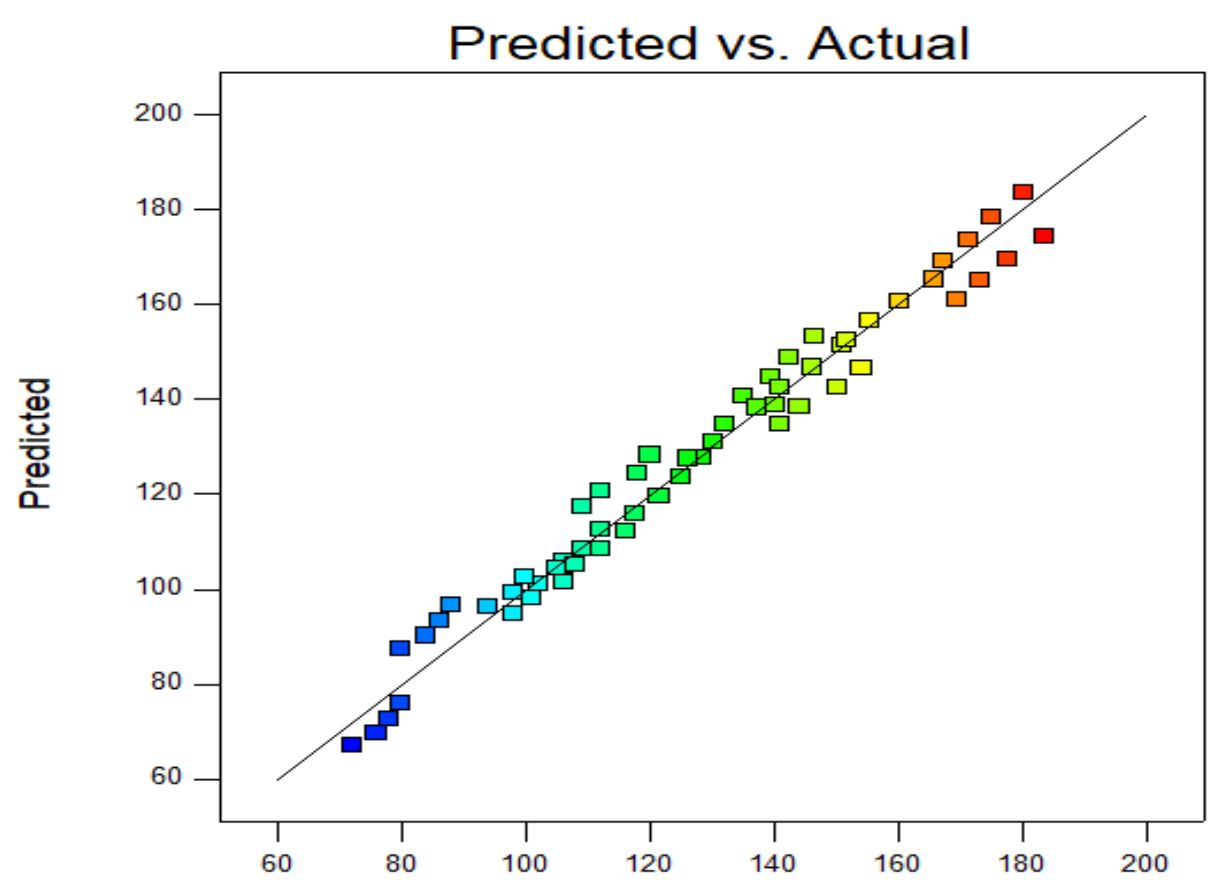

Actual

Fig. 7 


\subsection{3-D Surface}

The 3-D surface plot is a projection of the contour plot giving shape in addition to the color and contour.

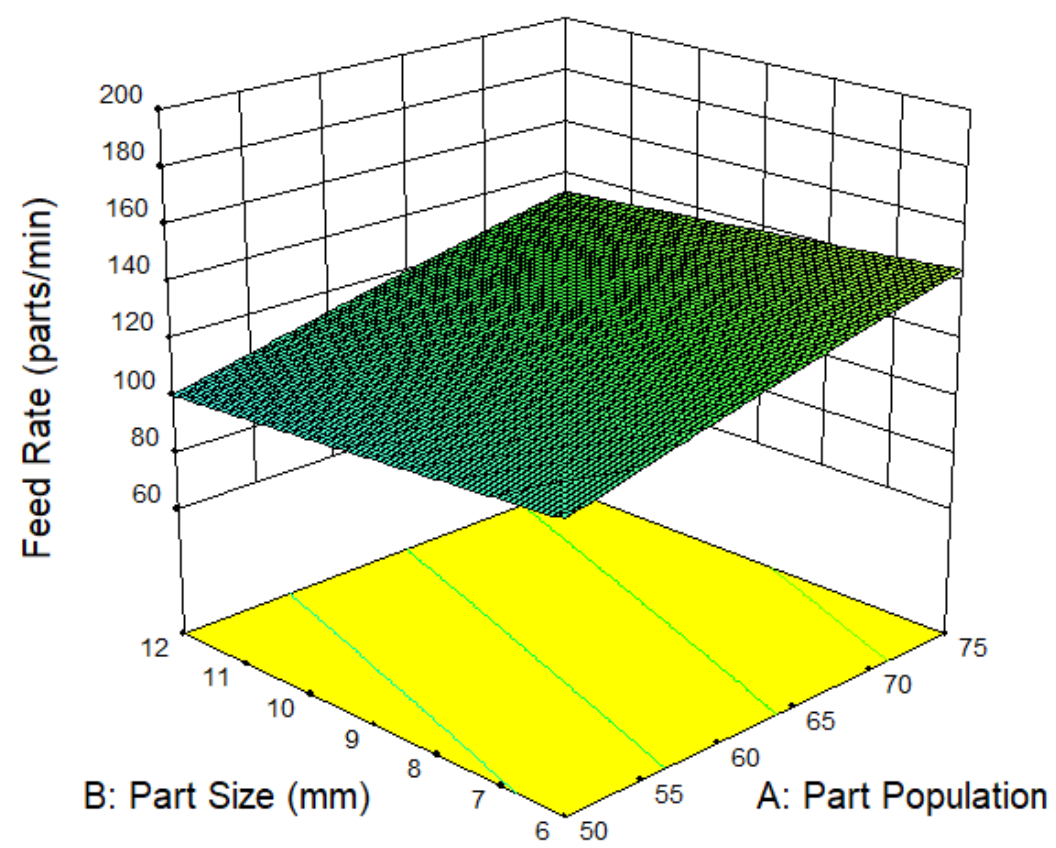

Fig. 8

\subsection{Cube Plot}

The cube plot shows the predicted values from the coded model for the combination of the lower and higher levels of any three factors.

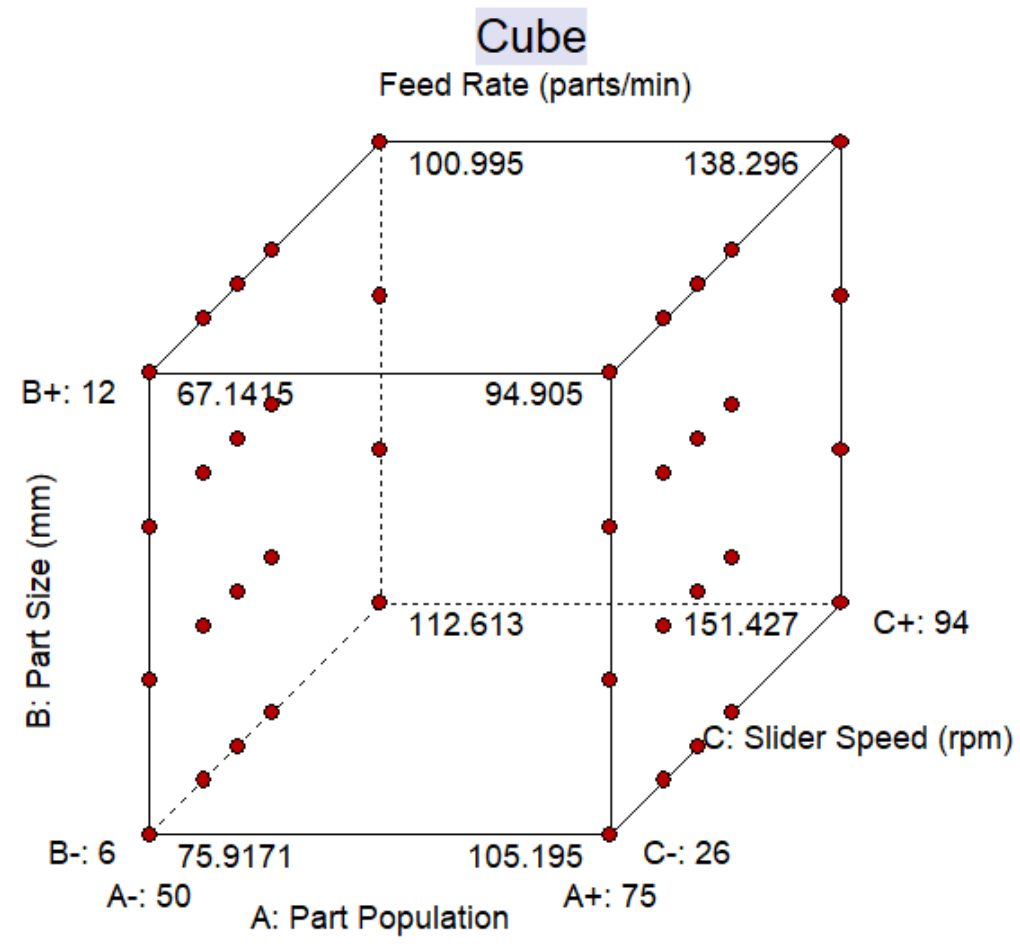

Fig. 9 


\subsection{Design Summary}

Design Summary

File Version 10.0.6.0

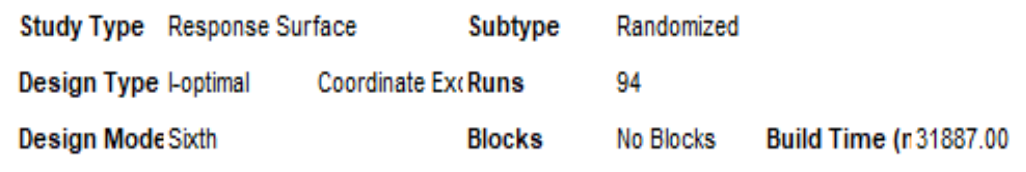

\begin{tabular}{lllllllllll} 
Factor & Name & Units & Type & Subtype & Minimum & Maximum & \multicolumn{2}{c}{ Coded Values } & Mean & Std. Dev. \\
A & Part Population & Numeric & Continuous & 0 & 100 & $-1.000=50$ & $1.000=75$ & 69.2676 & 19.8446 \\
B & Part Size & $\mathrm{mm}$ & Numeric & Continuous & 0 & 12 & $-1.000=6$ & $1.000=12$ & 8.82323 & 2.45043 \\
C & Slider Speed & $\mathrm{rpm}$ & Numeric & Continuous & 0 & 94 & $-1.000=26$ & $1.000=94$ & 54.1852 & 22.9613
\end{tabular}

$\begin{array}{llllllllllll}\text { Response } & \text { Name } & \text { Units } & \text { Obs } & \text { Analysis } & \text { Minimum } & \text { Maximum } & \text { Mean } & \text { Std. Dev. } & \text { Ratio } & \text { Trans } & \text { Model } \\ \text { R1 } & \text { Feed Rate } & \text { parts/min } & 60 & \text { Polynomial } & 71.9942 & 183.486 & 126.026 & 29.9154 & 2.54862 & \text { None } & \text { Quadratic }\end{array}$
Fig. 10

Model Summary Statistics

Source
Linear
$2 \mathrm{FI}$

\section{CONCLUSION}

An effort has been made to present an overview to a Mechanized Segment Feeder in order to optimize its use in the industry requiring small sized industrial components at given rate and orientation to encourage quality and uniform production rate. Results from the above graphs show that the designed system functions optimally at slider speed of 55-65 RPM with part population of about 100 of corresponding size of $8 \mathrm{~mm}$, giving a feed rate of 160 parts $/ \mathrm{min}$. However, the future work is required to show the simulation of results on actual feeders.

\section{Adjusted Predicted}

R-Squared R-Squared PRESS

$\begin{array}{lll}0.8937 & 0.8839 & 6132.07\end{array}$

$\begin{array}{lll}0.8967 & 0.8776 & 6464.94\end{array}$

$\underline{0.9695} \quad \underline{0.9649} \quad \underline{1855.84}$

$\begin{array}{lll}0.9987 & 0.9983 & 91.84\end{array}$

\section{Suqgested}

Aliased

Fig. 11

\section{REFERENCES}

[1]. Martin Maher, MSc,2010.”Design/Development of Automated Programmable Orientation of Tools for Vibratory Bowl Feeders ", Waterford institute of technology.

[2]. B.Mirtich, Y. Zhuang, K. Goldberg, John Craig, R. Zanutta, B. Carliste, J. Canny, " Estimating pose statistics for robotic part feeders", 1996, IEEE International Conference on Robotics and Automation, Minnesota.

[3]. Dina R. Berkowitz, John Canny, Department of electrical engineering, University of California. "Designing Parts Feeder Using Dynamic Simulation”, Berkeley. 
[4]. Kenneth Y. Goldberg, 1992. "Orienting polygonal parts without sensors". University Of South California, Los Angeles.

[5]. Loring J. Dohm, Lawrence B. Judge, Dwight M. Morgan, 1988."Method and system for the automated driving of parts and device used therein".

[6]. P. Pandey, Kushgra Varshneya, Pradeep Khanna, "Elevated Step Feeder Design, Fabrication and Study of Effects of Operating Parameters on Its Performance", 2016, IJETMAS.

[7]. W. Paul Cullen, Thomas J. Petronis, Clifford C. Annis, E. M. Ross, 1994, "Automated exchangeable parts feeding system".

[8]. Avinash Khatri, Mayank Sethi, Pradeep Khanna, 2016, "Development of a Flexible Elevated Gravimetric Part Feeding System with Performance Analysis". 